\title{
Proinflammatory cytokine levels are linked to death in pulmonary arterial hypertension
}

\begin{abstract}
To the Editor:
Recent data suggest that inflammatory processes play a major role in pulmonary vascular remodelling in patients with idiopathic, heritable, and drug- or toxin-induced (ihd) pulmonary arterial hypertension (PAH) $[1,2]$. Besides gaining insight into the mechanisms of PAH, understanding the link between inflammation and PAH may help to identify future therapeutic targets. Different studies have shown increased levels of cytokines in ihdPAH, including the proinflammatory cytokines interleukin (IL)-1 $\beta$, IL-2, IL-4, IL-6, IL- 8 , IL-12 ${ }^{\mathrm{p} 70}$, tumour necrosis factor (TNF)- $\alpha$, monocyte chemoattractant protein- $1[2,3]$, and the cytokine-like hormone leptin [4]. Advances in highly sensitive multiplex detection technologies present new opportunities to rapidly and specifically measure many cytokines using a limited sample volume [5]; the potential of combining markers rather than focusing on any single biomarker is of considerable interest $[3,6]$. Using such an approach, Soon et al. [7] recently suggested that IL-2, IL-6, IL-8, IL-10 and IL-12 $2^{\mathrm{p} 70}$ levels were associated with mortality in patients with ihdPAH. The French Network of Pulmonary Hypertension recently conducted a multicentre prospective cohort study of incident cases of PAH followed up for 3 years [8], from which a biobank of plasma stored as microstraws at $-180^{\circ} \mathrm{C}$ was available. Therefore, we tested our prospective cohort to determine whether cytokines quantified using a highly sensitive multiplex assay predict mortality in patients with ihdPAH.
\end{abstract}

Briefly, consecutive incident cases of PAH presenting at the University Pulmonary Vascular Departments in Clamart (Paris), Marseille, Grenoble, Strasbourg, Nancy and Toulouse (France) were enrolled between December 2003 and April 2006. Incident cases were defined as PAH patients with a diagnosis of PAH confirmed by right-heart catheterisation, without current or previous treatment with drugs approved for the management of PAH such as endothelin receptor antagonists, type 5 phosphodiesterase inhibitors or prostacyclins.

For each patient, 24-mL venous blood samples taken during initial diagnostic right-heart catheterisation using the central venous line were anonymised and immediately transferred to the Annemasse French Blood Service biobank, where EDTA plasma and serum microstraws were stored at $-180^{\circ} \mathrm{C}$. In February 2011, EDTA plasma microstraws, available from 74 patients with ihdPAH, were sent via an express carrier on dry ice to Grenoble University Hospital, France for cytokine quantification. Cytokines were quantified using Randox multiplex assays (Randox Laboratories, Crumlin, UK), using cytokine arrays I and V. The cytokines quantified are listed in table 1. Death and cause of death were verified from hospital records, contact with primary care physicians and, when required, using the INSERM French Mortality Registry. The primary study outcome was all-cause mortality.

Patients were censored at 3 years or at time of lung or heart/lung transplantation. We used univariable Cox proportional hazards models to analyse the unadjusted association between baseline characteristics and allcause mortality. We estimated the adjusted hazard ratios (HRs) for death and their corresponding $95 \%$ confidence intervals using a multivariable Cox proportional hazards model. p-values of $<0.05$ were considered statistically significant. All analyses were performed using Stata version 11 (Stata Corporation, College Station, TX, USA).

The analysed sample consisted of 74 patients with ihdPAH. The mean \pm SD age of all patients was $51.5 \pm 17.5$ years and $38(51.3 \%)$ were male. Baseline patient characteristics have been reported previously [8] and plasma cytokine levels are presented in table 1.

17 patients died within 3 years of enrolment. In univariable analysis, the baseline characteristics associated with increased hazard of death included New York Heart Association class IV (HR 17, 95\% CI 1.75-164; $\mathrm{p}=0.01$ ) and lower values for the 6-min walk distance (HR 0.41, 95\% CI 0.24-0.69, for 1-SD increment; $\mathrm{p}=0.001$ ) while a nonsignificant trend towards greater hazard of death was observed for age (HR 1.69, 95\%

For editorial comments see page 685 . 
TABLE 1 Baseline plasma cytokine levels and associated hazard ratios (HRs) for death for patients with idiopathic, heritable, and drug- and toxin-induced pulmonary arterial hypertension

\begin{tabular}{|c|c|c|c|c|c|}
\hline Cytokine & Concentration $\mathrm{pg} \cdot \mathrm{mL}^{-1}$ & Unadjusted HR $(95 \% \mathrm{CI})^{\#}$ & p-value & Adjusted HR $(95 \% \mathrm{CI})^{\top}$ & $\mathrm{p}$-value \\
\hline IL-1 $\alpha$ & $0.52 \pm 1.11$ & $1.35(1.01-1.82)$ & 0.04 & $1.74(1.25-2.43)$ & 0.001 \\
\hline IL-3 & $4.07 \pm 5.75$ & $1.42(0.89-2.26)$ & 0.14 & & \\
\hline IL-4 & $1.24 \pm 1.38$ & $1.04(0.65-1.67)$ & 0.87 & & \\
\hline IL-6 & $3.64 \pm 5.21$ & $1.45(1.09-1.92)$ & 0.01 & $1.33(0.98-1.81)$ & 0.06 \\
\hline IL-10 & $1.65 \pm 10.67$ & $0.71(0.12-4.24)$ & 0.71 & & \\
\hline IL-12 $2^{\text {p70 }}$ & $2.64 \pm 3.90$ & $0.96(0.59-1.57)$ & 0.89 & & \\
\hline IL-13 & $1.28 \pm 1.93$ & $1.54(1.00-2.38)$ & 0.05 & $1.81(1.08-3.01)$ & 0.02 \\
\hline IL-23 & $5 \pm 6$ & $1.44(0.57-3.66)$ & 0.44 & & \\
\hline IFN- $\gamma$ & $0.72 \pm 1.10$ & $1.09(0.68-1.76)$ & 0.72 & & \\
\hline TNF- $\alpha$ & $3.07 \pm 1.68$ & $1.50(1.14-1.96)$ & 0.004 & $1.69(1.14-2.51)$ & 0.009 \\
\hline
\end{tabular}

Data are presented as mean $\pm S D$, unless otherwise stated. $n=74$. IL: interleukin; IFN: interferon; TNF: tumour necrosis factor; MCP: monocyte chemoattractant protein; VEGF: vascular endothelial growth factor; EGF: epidermal growth factor. \#: corresponds to 1 SD increment in the values except for IL-23, for which the HR corresponds to the detection of values $>0.1 \mathrm{ng} \cdot \mathrm{mL}^{-1}$. ${ }^{\circ}$ : adjusted for age, 6 -min walk distance, cardiac output and right atrial pressure; missing values for covariates were imputed by means of multiple imputations.

CI $0.97-2.94 ; \mathrm{p}=0.06$ ) and right atrial pressure (HR 1.56, 95\% CI 0.94-2.59, $\mathrm{p}=0.08$ ). The proinflammatory cytokines IL- $\alpha$, IL-1 $\beta$, IL-6, TNF- $\alpha$ and IL-13 were associated with an increased unadjusted hazard of death (table 1). After adjusting for age, 6-min walk distance, cardiac output, and right atrial pressure, higher levels of proinflammatory cytokines IL- $1 \alpha$, IL- $1 \beta$, TNF- $\alpha$ and IL-13 remained independently associated with mortality, while IL-6 was associated with a nonsignificant trend towards increased hazard of death (table 1).

Therefore, we confirm previous reports showing that the levels of various proinflammatory cytokines can predict mortality in a homogenous group of patients with ihdPAH. While we observed differences in terms of cytokine prognosis, IL-6 consistently predicts poor outcome in the three available studies [7, 9].

It remains unclear how inflammation contributes to the pathogenesis of PAH. It is likely that cytokines are produced as a result of an initial activation of adaptive immunity to further propagate the inflammatory process on their own or through the production of growth factors [2]. However, we did not observe any association between growth factor levels and mortality. The observed link between cytokine levels and mortality does not imply that the blockade of a specific cytokine receptor will improve patient outcome. Indeed, the whole range of inflammatory cytokines seems to be activated and further physiopathological investigation is required to determine whether one specific pathway may be prominent. Furthermore, this link provides a rationale for targeting inflammation, as recently performed in animal models where dexamethasone reversed monocrotaline-induced pulmonary hypertension in rats [10]. Such an approach could be tested using IL-6 receptor blockers such as tocilizumab.

The main limitation of our study relates to the interval between plasma sampling and cytokine quantification. When considering cytokine measurement, several issues should be considered in order to maintain cytokine stability: 1$)$ samples should be stored at low temperatures $\left.\left(\leqslant-80^{\circ} \mathrm{C}\right) ; 2\right)$ multiple freezethaw cycles should be avoided; and 3) while most cytokines remain stable within a 2-year period, longer term storage may alter cytokine levels as a result of cross-reactivity between protein epitopes [11]. Despite the fact that samples were kept at $-180^{\circ} \mathrm{C}$ and they did not undergo any freeze-thaw cycles, as used samples do not re-enter the biobank, the storage time was long (5-8 years). Therefore, we cannot exclude cytokine degradation and we could not test it earlier given that routine multiplex analysis was unavailable at the time of study conception. This may explain why our levels of IL-6, IL-10, IL-12, IL-13 and TNF- $\alpha$ were lower than those of Soon et al. [7]. A second potential limitation is the high male to female ratio. The most plausible explanation is that it may be due to chance in a small cohort. The second potential explanation is 
that being female is a strong positive prognostic factor [12], implying that cohorts enrolling incident cases are more likely to enrol men than cohorts based on prevalent cases.

In conclusion, our data further support the concept that proinflammatory cytokines are independently associated with mortality in patients with $\mathrm{PAH}$, emphasise the possible role of inflammation in PAH and support the interest in targeting inflammation when developing future $\mathrm{PAH}$ therapeutic strategies. The current routine availability of multiplex assays may also prompt proinflammatory cytokine quantification in order to help refine patients' prognoses at the time of diagnostic right-heart catheterisation. arterial hypertension http://ow.ly/qOacN

Jean-Luc Cracowski ${ }^{1,2}$, François Chabot ${ }^{3}$, José Labarère ${ }^{4,5}$, Patrice Faure ${ }^{2,6}$, Bruno Degano ${ }^{7}$, Carole Schwebel ${ }^{8}$, Ari Chaouat ${ }^{3}$, Martine Reynaud-Gaubert ${ }^{9}$, Claire Cracowski ${ }^{1}$, Olivier Sitbon ${ }^{10,11,12}$, Azzedine Yaici ${ }^{10,11,12}$, Gerald Simonneau ${ }^{10,11,12}$ and Marc Humbert ${ }^{10,11,12}$

${ }^{1}$ Clinical Pharmacology Dept, INSERM CIC3, University Hospital, Grenoble, ${ }^{2}$ University Grenoble Alpes, INSERM U1042, Grenoble, ${ }^{3}$ Pulmonology Dept, University Hospital, Nancy, ${ }^{4}$ TIMC UMR 5525 CNRS, University Grenoble Alpes, Grenoble, ${ }^{5}$ Quality of Care Unit, University Hospital, Grenoble, ${ }^{6}$ Dept of Integrated Biology, University Hospital, Grenoble, ${ }^{7}$ Physiology Dept, University Hospital, Besançon, ${ }^{8}$ Intensive Care Unit, University Hospital, Grenoble, ${ }^{9}$ Pulmonology Dept, University Hospital, Marseille, ${ }^{10}$ University Paris-Sud, Medical Faculty, Le Kremlin-Bicêtre, ${ }^{11}$ AP-HP, Pulmonology Dept, Thorax Innovation Hospital-University Dept (DHU TORINO), Hôpital Bicêtre, Le Kremlin-Bicêtre, and ${ }^{12}$ INSERM U999, LabEx LERMIT, Marie Lannelongue Surgical Centre, Le Plessis Robinson, France.

Correspondence: J-L. Cracowski, Unité de Pharmacologie Clinique, INSERM CIC3, Grenoble University Hospital, CS10217, 38043 Grenoble Cedex 09, France. E-mail: Jean-Luc.Cracowski@ujf-grenoble.fr

Received: Aug 302013 | Accepted after revision: Nov 032013 | First published online: Nov 142013

Support statement: The study was supported by the French Health Ministry, the French "Institut National pour la santé et la Recherche Médicale" (INSERM), and by unrestricted research grants from AGIRàdom, Pneumologie Developpement and Pfizer.

Conflict of interest: Disclosures can be found alongside the online version of this article at www.erj.ersjournals.com

Acknowledgements: We thank N. Gonnet (Grenoble Clinical Research Centre, Grenoble, France) for his major role in managing the project and ensuring quality control and data management. A. Foote (Grenoble Clinical Research Centre, Grenoble, France) edited the manuscript for English usage.

\section{References}

1 Voelkel NF, Gomez-Arroyo J, Abbate A, et al. Pathobiology of pulmonary arterial hypertension and right ventricular failure. Eur Respir J 2012; 40: 1555-1565.

2 Price LC, Wort SJ, Perros F, et al. Inflammation in pulmonary arterial hypertension. Chest 2012; 141: 210-221.

3 Cracowski JL, Leuchte HH. The potential of biomarkers in pulmonary arterial hypertension. Am J Cardiol 2012; 110: Suppl. 6, 32S-38S.

4 Huertas A, Tu L, Gambaryan N, et al. Leptin and regulatory T-lymphocytes in idiopathic pulmonary arterial hypertension. Eur Respir J 2012; 40: 895-904.

5 Zhou X, Fragala MS, McElhaney JE, et al. Conceptual and methodological issues relevant to cytokine and inflammatory marker measurements in clinical research. Curr Opin Clin Nutr Metab Care 2010; 13: 541-547.

6 Cracowski JL. Towards prognostic biomarkers in pulmonary arterial hypertension. Eur Respir J 2012; 39: 799-801.

7 Soon E, Holmes AM, Treacy CM, et al. Elevated levels of inflammatory cytokines predict survival in idiopathic and familial pulmonary arterial hypertension. Circulation 2010; 122: 920-927.

8 Cracowski JL, Degano B, Chabot F, et al. Independent association of urinary F2-isoprostanes with survival in pulmonary arterial hypertension. Chest 2012; 142: 869-876.

9 Rhodes CJ, Wharton J, Howard LS, et al. Red cell distribution width outperforms other potential circulating biomarkers in predicting survival in idiopathic pulmonary arterial hypertension. Heart 2011; 97: 1054-1060.

10 Price LC, Montani D, Tcherakian C, et al. Dexamethasone reverses monocrotaline-induced pulmonary arterial hypertension in rats. Eur Respir J 2011; 37: 813-822.

11 Keustermans GCE, Hoeks SBE, Meerding JM, et al. Cytokine assays: an assessment of the preparation and treatment of blood and tissue samples. Methods 2013; 61: 10-17.

12 Humbert M, Sitbon O, Chaouat A, et al. Survival in patients with idiopathic, familial, and anorexigen-associated pulmonary arterial hypertension in the modern management era. Circulation 2010; 122: 156-163. 